\title{
The Multivariate Order Statistics for Exponential and Weibull Distributions
}

\author{
Mariyam Hafeez \\ Department of Statistics \\ Lahore College for Women University \\ Lahore, Pakistan \\ ume_mariyam@hotmail.com \\ Shahid Kamal \\ College of Statistical and Actuarial Sciences \\ University of the Punjab \\ Lahore, Pakistan \\ kamal_shahid@hotmail.com \\ Muhammad Qaiser Shahbaz \\ Department of Statistics \\ COMSATS Institute of IT \\ Lahore, Pakistan \\ drmqshahbaz@ciitlahore.edu.pk
}

\begin{abstract}
In this paper we have derived the distribution of multivariate order statistics for multivariate exponential \& multivariate weibull distribution. The moment expression for multivariate order statistics has also been derived.
\end{abstract}

Keywords: Multivariate exponential distribution, Multivariate Weibull distribution, Order Statistics, Moments.

\section{Introduction:}

Let $X_{1}, X_{2}, \ldots, X_{p}$ be a set of $\mathrm{p}$ random variables with density function $f_{i}\left(x_{i}\right)$ and distribution function $F_{i}\left(x_{i}\right)$. Gumbel (1958) provided following method of obtaining joint density of the vector $\mathbf{x}$ as:

$$
f(\mathbf{x})=\prod_{i=1}^{p} f_{i}\left(x_{i}\right)\left[1+\theta \prod_{i=1}^{p}\left\{2 F_{i}\left(x_{i}\right)-1\right\}\right]
$$

Suppose $\mathrm{Y}$ is another random variable with density function $f(y)$ and distribution function $F(y)$ then the joint density of $\mathrm{Y}$ and $\mathbf{x}$ may be obtained by using:

$$
f(y, \mathbf{x})=f(y) \times \prod_{i=1}^{p} f_{i}\left(x_{i}\right)\left[1+\theta\{2 F(y)-1\} \times \prod_{i=1}^{p}\left\{2 F_{i}\left(x_{i}\right)-1\right\}\right]
$$

which can be used to generate multivariate distributions based on any available marginal. Several authors have used (1.2) to propose multivariate distributions. 
Order Statistics has been extensively studied by many authors. Specifically, the distribution of $r$ th order statistics as given in David and Nagaraja (2003) is

$$
f_{(r: n)}(x)=\frac{n !}{(r-1) !(n-r) !} f(x)[F(x)]^{r-1}[1-F(x)]^{n-r} ;
$$

where $F(x)$ is distribution function of the parent distribution.

The univariate order statistics for special distributions has been extensively studied by several researchers in context of their application in extreme events. The order statistics has also been used to study the concomitants for bivariate distribution as discussed by Veena and Thomas (2008).

The multivariate order statistics has been area of researcher in recent years. Belzunce et al (2003) has presented the idea of multivariate order statistics but not with much applicability. Barakat (2009) provided some results for multivariate order statistics for dependent random variables. Arnold (2009) has developed the density of multivariate order statistics of a random vector $\mathbf{X}$ by using the idea of concomitants. The density of multivariate order statistics is obtained by using:

$$
f_{(r: n)}(\mathbf{x})=\frac{n !}{(r-1) !(n-r) !} f(\mathbf{x}) \int_{-\infty}^{\infty}[F(y)]^{r-1}[1-F(y)]^{n-r} f(y \mid \mathbf{x}) d y
$$

where $f(y \mid \mathbf{x})$ is the conditional distribution of Y given $\mathbf{x}$. Hafeez et al (2011) discussed the bivariate order statistics for exponential distribution by using (1.3).

In this paper we have studied the multivariate order statistics for exponential and Weibull distribution. These distributions have been discussed in the following sections.

We first define a multivariate exponential distribution by using (1.2). Suppose that every component of vector $\mathbf{x}$ has common Exponential Distribution with density and distribution function given as:

$$
f_{i}\left(x_{i}\right)=\alpha_{i} e^{-\alpha_{i} x_{i}} \quad \text { and } \quad F_{i}\left(x_{i}\right)=1-e^{-\alpha_{i} x_{i}} ;
$$

Further, suppose that the random variable $Y$ also has Exponential distribution with density and distribution function as:

$$
f(y)=\beta e^{-\beta y} \text { and } F(y)=1-e^{-\beta y} .
$$

The joint density of $\mathrm{Y}$ and $\mathbf{x}$ is obtained by using (5.2.2) as:

$$
f(y, \mathbf{x})=\beta k e^{-\beta y} \exp \left(-\sum_{i=1}^{p} \alpha_{i} x_{i}\right)\left[1+\theta\left(1-2 e^{-\beta y}\right) \prod_{i=1}^{p}\left\{1-2 e^{-\alpha_{i} x_{i}}\right\}\right]
$$

where $k=\prod_{i=1}^{p} \alpha_{i}$

The joint marginal distribution of $\mathbf{x}$ is obtained from (5.2.4) as: 


$$
\begin{aligned}
f(\mathbf{x}) & =\int_{0}^{\infty} \beta k e^{-\beta y} \exp \left(-\sum_{i=1}^{p} \alpha_{i} x_{i}\right)\left[1+\theta\left(1-2 e^{-\beta y}\right) \prod_{i=1}^{p}\left\{1-2 e^{-\alpha_{i} x_{i}}\right\}\right] d y \\
& =k \exp \left(-\sum_{i=1}^{p} \alpha_{i} x_{i}\right) \int_{0}^{\infty} \beta e^{-\beta y}\left[1+\theta\left(1-2 e^{-\beta y}\right) \prod_{i=1}^{p}\left\{1-2 e^{-\alpha_{i} x_{i}}\right\}\right] d y \\
& =k \exp \left(-\sum_{i=1}^{p} \alpha_{i} x_{i}\right)
\end{aligned}
$$

The conditional distribution of $\mathrm{Y}$ given $\mathbf{x}$ is:

$$
\begin{aligned}
& f(y \mid \mathbf{x})=\frac{f(y, \mathbf{x})}{f(\mathbf{x})} \\
& f(y \mid \mathbf{x})=\frac{\beta k e^{-\beta y} \exp \left(-\sum_{i=1}^{p} \alpha_{i} x_{i}\right)\left[1+\theta\left(1-2 e^{-\beta y}\right) \prod_{i=1}^{p}\left\{1-2 e^{-\alpha_{i} x_{i}}\right\}\right]}{k \exp \left(-\sum_{i=1}^{p} \alpha_{i} x_{i}\right)} \\
& f(y \mid \mathbf{x})=\beta e^{-\beta y}\left[1+\theta\left(1-2 e^{-\beta y}\right) \prod_{i=1}^{p}\left\{1-2 e^{-\alpha_{i} x_{i}}\right\}\right]
\end{aligned}
$$

The distribution of $\mathrm{r}^{\text {th }}$ multivariate order statistics is derived in the following section.

\section{2. $\mathbf{R}^{\text {th }}$ Multivariate Order Statistics for Exponential Distribution}

In this section the $\mathrm{r}^{\text {th }}$ multivariate order statistics for exponential distribution has been derived. The density of $\mathrm{r}^{\text {th }}$ multivariate order statistics is obtained by using (5.2.5) in (5.2.2) as:

$$
\begin{aligned}
f_{r: n}(\underline{x})= & \frac{n !}{(r-1) !(n-r) !} f(x) \int_{-\infty}^{\infty} f(y / x)[F(y)]^{r-1}[1-F(y)]^{n-r} d y \\
f_{r: n}(\underline{x})= & \frac{n !}{(r-1) !(n-r) !} k \exp \left(-\sum_{i=1}^{p} \alpha_{i} x_{i}\right) \int_{0}^{\infty}\left(1-e^{-\beta y}\right)^{r-1}\left(e^{-\beta y}\right)^{n-r} \beta e^{-\beta y}\left[1+\theta\left(1-2 e^{-\beta y}\right)\right. \\
& \left.\prod_{i=1}^{p}\left(1-2 e^{-\alpha_{i} x_{i}}\right)\right] d y \\
f_{r: n}(\underline{x})= & \frac{n !}{(r-1) !(n-r) !} k \exp \left(-\sum_{i=1}^{p} \alpha_{i} x_{i}\right) \frac{(n-r) !(r-1) !}{n !}+\theta \prod_{i=1}^{p}\left(1-2 e^{-\alpha_{i} x_{i}}\right) \\
{\left[\int_{0}^{\infty} \beta e^{-\beta y}\left(1-e^{-\beta y}\right)\right.} &
\end{aligned}
$$




$$
\begin{aligned}
& f_{r: n}(\underline{x})=\frac{n !}{(r-1) !(n-r) !} k \exp \left(-\sum_{i=1}^{p} \alpha_{i} x_{i}\right) \frac{(n-r) !(r-1) !}{n !}\left[1+\theta \prod_{i=1}^{p}\left(1-2 e^{-\alpha_{i} x_{i}}\right)\left(\frac{2 r}{n+1}-1\right)\right] \\
& f_{r: n}(\underline{x})=k \exp \left(-\sum_{i=1}^{p} \alpha_{i} x_{i}\right)\left[1+\theta \prod_{i=1}^{p}\left(1-2 e^{-\alpha_{i} x_{i}}\right)\left(\frac{2 r-n-1}{n+1}\right)\right]
\end{aligned}
$$

where $k=\prod_{i=1}^{p} \alpha_{i}$

The moments of $\mathrm{r}^{\text {th }}$ multivariate order statistics are derived in the following section.

\section{Moments for Multivariate Order Statistics for Exponential Distribution}

In this section the Moments for $\mathrm{r}^{\text {th }}$ multivariate order statistics has been derived. The $\mathrm{k}^{\text {th }}$ joint moment of a set of random variables is given as:

$$
E\left(X_{1 r: n}^{k}, X_{2 r: n}^{k}, \ldots . . X_{p r: n}^{k}\right)=\int_{0}^{\infty} \int_{0}^{\infty} \ldots \ldots . \int_{0}^{\infty} x_{1}^{k} x_{2}^{k} \ldots \ldots . x_{p}^{k} f_{r ; n}\left(x_{1}, x_{2}, \ldots . x_{p}\right) d x_{1} d x_{2} \ldots . d x_{p}
$$

Using (5.3.2) in (5.4.1), the moments are given as:

$$
\begin{aligned}
& E\left(X_{1 r: n}^{k}, X_{2 r: n}^{k}, \ldots \ldots . X_{p r: n}^{k}\right)=\int_{0}^{\infty} \int_{0}^{\infty} \ldots \ldots . \int_{0}^{\infty} x_{1}^{k} x_{2}^{k} \ldots \ldots x_{p}^{k} k \exp \left(-\sum_{i=1}^{p} \alpha_{i} x_{i}\right) \\
& {\left[1+\theta \prod_{i=1}^{p}\left(1-2 e^{-\alpha_{i} x_{i}}\right)\left(\frac{2 r-n-1}{n+1}\right)\right] d x_{1} d x_{2} \ldots . d x_{p}} \\
& E\left(X_{1 r: n}^{k}, X_{2 r: n}^{k}, \ldots \ldots X_{p r: n}^{k}\right)=\int_{0}^{\infty} x_{1}^{k} \alpha_{1} e^{-\alpha_{1} x_{1}} d x_{1} \int_{0}^{\infty} x_{2}^{k} \alpha_{2} e^{-\alpha_{2} x_{2}} d x_{2} \ldots . . \int_{0}^{\infty} x_{p}^{k} \alpha_{p} e^{-\alpha_{p} x_{p}} d x_{p} \\
& +\theta\left(\frac{2 r-n-1}{n+1}\right) \int_{0}^{\infty} x_{1}^{k} \alpha_{1} e^{-\alpha_{1} x_{1}}\left(1-2 e^{-\alpha_{1} x_{1}}\right) d x_{1} \int_{0}^{\infty} x_{2}^{k} \alpha_{2} e^{-\alpha_{2} x_{2}}\left(1-2 e^{-\alpha_{2} x_{2}}\right) d x_{2} \\
& \times \ldots \ldots . . \times \int_{0}^{\infty} x_{p}^{k} \alpha_{p} e^{-\alpha_{p} x_{2 p}}\left(1-2 e^{-\alpha_{p} x_{p}}\right) d x_{p}
\end{aligned}
$$

Or

$$
\begin{gathered}
E\left(X_{1 r: n}^{k}, X_{2 r: n}^{k}, \ldots \ldots X_{p r: n}^{k}\right)=\prod_{t=1}^{p} I_{t}+\theta\left(\frac{2 r-n-1}{n+1}\right) \prod_{t=1}^{p} M_{t} \\
\text { where } I_{t}=\int_{0}^{\infty} x_{t}^{k} \alpha_{t} e^{-\alpha_{t} x_{t}} d x_{t} ; \quad t=1,2, \ldots, p \\
M_{t}=\int_{0}^{\infty} x_{t}^{k} \alpha_{t} e^{-\alpha_{t} x_{t}}\left(1-2 e^{-\alpha_{t} x_{t}}\right) d x_{t} ; \quad t=1,2, \ldots, p
\end{gathered}
$$


Consider: $I_{t}=\int_{0}^{\infty} x_{t}^{k} \alpha_{t} e^{-\alpha_{t} x_{t}} d x_{t}$

$$
\begin{array}{r}
\text { Let } w=\alpha_{t} x_{t} \Rightarrow d w=\alpha_{t} d x_{t} ; x_{t}=\frac{w}{\alpha_{t}} \\
I_{t}=\frac{1}{\alpha_{t}^{k}} \int_{0}^{\infty} w^{k} e^{-w} d w=\frac{1}{\alpha_{t}^{k}} \Gamma(k+1)
\end{array}
$$

Now consider $M_{t}$ as:

$$
\begin{aligned}
& M_{t}=\int_{0}^{\infty} x_{t}^{k} \alpha_{t} e^{-\alpha_{t} x_{t}}\left(1-2 e^{-\alpha_{t} x_{t}}\right) d x_{t} \\
& M_{1}=\frac{1}{\alpha_{t}^{k}} \int_{0}^{\infty} w_{t}^{k} e^{-w_{t}}\left(1-2 e^{-w_{t}}\right) d w ; \text { with } w_{t}=\alpha_{t} x_{t} \\
& M_{t}=\frac{\Gamma(k+1)}{\alpha_{t}^{k}}\left(\frac{2^{k}-1}{2^{k}}\right)
\end{aligned}
$$

Using values of $I_{1}, I_{2}, \ldots . . I_{p} \& M_{1}, M_{2}, \ldots . . M_{p}$ in (5.4.2) we have:

$$
\begin{aligned}
E\left(X_{1 r: n}^{k}, X_{2 r: n}^{k}, \ldots \ldots X_{p r: n}^{k}\right) & =\frac{1}{\alpha_{1}^{k}} \sqrt{k+1} \frac{1}{\alpha_{2}^{k}} \sqrt{k+1} \ldots \ldots . . \frac{1}{\alpha_{p}^{k}} \sqrt{k+1}+\theta\left(\frac{2 r-n-1}{n+1}\right) \\
& \frac{\sqrt{k+1}}{\alpha_{1}^{k}} \frac{\left(2^{k}-1\right)}{\left(2^{k}\right)} \cdot \frac{\sqrt{k+1}}{\alpha_{2}^{k}} \frac{\left(2^{k}-1\right)}{\left(2^{k}\right)} \ldots \frac{\sqrt{k+1}}{\alpha_{p}^{k}} \frac{\left(2^{k}-1\right)}{\left(2^{k}\right)} \\
E\left(X_{1 r: n}^{k}, X_{2 r: n}^{k}, \ldots \ldots X_{p r: n}^{k}\right)= & \prod_{i=1}^{p} \frac{(\sqrt{k+1})}{\alpha_{i}^{k}}+\prod_{i=1}^{p} \frac{(\sqrt{k+1})}{\alpha_{i}^{k}} \frac{\left(2^{k}-1\right)}{\left(2^{k}\right)} \theta\left(\frac{2 r-n-1}{n+1}\right) \\
E\left(X_{1 r: n}^{k}, X_{2 r: n}^{k}, \ldots \ldots . X_{p r: n}^{k}\right)= & \frac{(\sqrt{k+1})^{p}}{\prod_{i=1}^{p} \alpha_{i}^{k}}+\frac{(\sqrt{k+1})^{p}}{\prod_{i=1}^{p} \alpha_{i}^{k}}\left\{\theta\left(\frac{2 r-n-1}{n+1}\right)\left(\frac{2^{k}-1}{2^{k}}\right)\right\}
\end{aligned}
$$

The moments of specific order can be obtained from (3.3).

\section{Multivariate Order Statistics for Weibull Distribution}

In this section the multivariate order statistics has been discussed. For this suppose that every component of vector $\mathbf{x}$ has common Weibull Distribution with density and distribution function given as:

$$
f_{i}\left(x_{i}\right)=\alpha_{i} x^{\alpha_{i}-1} \exp \left(-x_{i}^{\alpha_{i}}\right) \quad \text { and } \quad F_{i}\left(x_{i}\right)=1-\exp \left(-x_{1}^{\alpha_{1}}\right)
$$


Further, suppose that the random variable $\mathrm{Y}$ also has weibull distribution with density and distribution function as:

$$
f(y)=\beta y^{\beta-1} \exp \left(-y^{\beta}\right) \quad \text { and } \quad F(y)=1-\exp \left(-y^{\beta}\right) .
$$

The joint density of $\mathrm{Y}$ and $\mathbf{x}$ is obtained by using (1.1) as:

$$
f(y, \mathbf{x})=\beta k e^{-\beta y} \exp \left(-\sum_{i=1}^{p} \alpha_{i} x_{i}\right)\left[1+\theta\left(1-2 e^{-\beta y}\right) \prod_{i=1}^{p}\left\{1-2 e^{-\alpha_{i} x_{i}}\right\}\right]
$$

where $k=\prod_{i=1}^{p} \alpha_{i}$

The joint marginal distribution of $\mathbf{x}$ is obtained from (4) as:

$$
\begin{aligned}
& f(\mathbf{x})=\int_{0}^{\infty} \beta k e^{-\beta y} \exp \left(-\sum_{i=1}^{p} \alpha_{i} x_{i}\right)\left[1+\theta\left(1-2 e^{-\beta y}\right) \prod_{i=1}^{p}\left\{1-2 e^{-\alpha_{i} x_{i}}\right\}\right] d y \\
& f(\mathbf{x})=k \exp \left(-\sum_{i=1}^{p} \alpha_{i} x_{i}\right) \int_{0}^{\infty} \beta e^{-\beta y}\left[1+\theta\left(1-2 e^{-\beta y}\right) \prod_{i=1}^{p}\left\{1-2 e^{-\alpha_{i} x_{i}}\right\}\right] d y \\
& f(\mathbf{x})=k \exp \left(-\sum_{i=1}^{p} \alpha_{i} x_{i}\right)
\end{aligned}
$$

The conditional distribution of $\mathrm{Y}$ given $\mathbf{x}$ is:

$$
\begin{aligned}
& f(y \mid \mathbf{x})=\frac{f(y, \mathbf{x})}{f(\mathbf{x})} \\
& f(y \mid \mathbf{x})=\frac{\beta k e^{-\beta y} \exp \left(-\sum_{i=1}^{p} \alpha_{i} x_{i}\right)\left[1+\theta\left(1-2 e^{-\beta y}\right) \prod_{i=1}^{p}\left\{1-2 e^{-\alpha_{i} x_{i}}\right\}\right]}{k \exp \left(-\sum_{i=1}^{p} \alpha_{i} x_{i}\right)} \\
& f(y \mid \mathbf{x})=\beta e^{-\beta y}\left[1+\theta\left(1-2 e^{-\beta y}\right) \prod_{i=1}^{p}\left\{1-2 e^{-\alpha_{i} x_{i}}\right\}\right]
\end{aligned}
$$

\section{5. $\mathbf{R}^{\text {th }}$ Multivariate Order Statistics for Trivariate Weibull Distribution}

In this section the $\mathrm{r}^{\text {th }}$ multivariate order statistics for multivariate weibull distribution has been derived. The density of $r^{\text {th }}$ multivariate order statistics is obtained by using (4.2) in (1.2) as:

$$
f(y / x)=\beta y^{\beta-1} e^{-y^{\beta}}\left[1+\theta\left(1-2 e^{-y^{\beta}}\right) \prod_{i=1}^{p}\left(1-2 e^{-x_{i}^{\alpha_{i}}}\right)\right]
$$




$$
\begin{gathered}
f_{r: n}(\underline{x})=\frac{n !}{(r-1) !(n-r) !} f(x) \int_{-\infty}^{\infty} f(y / x)[F(y)]^{r-1}[1-F(y)]^{n-r} d y \\
f_{r: n}(\underline{x})=\frac{n !}{(r-1) !(n-r) !} k \prod_{i=1}^{p} x_{i}^{\alpha_{i}-1} \exp \left(-\sum_{i=1}^{p} x_{i}^{\alpha_{i}}\right) \\
\int_{0}^{\infty} \beta y^{\beta-1} e^{-y^{\beta}}\left(1-e^{-y^{\beta}}\right)^{r-1}\left(e^{-y^{\beta}}\right)^{n-r}\left(1+\theta\left(1-2 e^{-y^{\beta}}\right)\right) \prod_{i=1}^{p}\left(1-2 e^{-x_{i}^{\alpha_{i}}}\right) d y \\
f_{r: n}(\underline{x})=\frac{n !}{(r-1) !(n-r) !} k \prod_{i=1}^{p} x_{i}^{\alpha_{i}-1} \exp \left(-\sum_{i=1}^{p} x_{i}^{\alpha_{i}}\right)\left[\frac{(n-r) !(r-1) !}{n !}+\theta \prod_{i=1}^{p}\left(1-2 e^{-x_{i}^{\alpha_{i}}}\right)\right. \\
\left\{\int_{0}^{\infty} \beta y^{\beta-1} e^{-y^{\beta}}\left(1-e^{-y^{\beta}}\right)^{r-1}\left(e^{-y^{\beta}}\right)^{n-r} d y-2 \int_{0}^{\infty} \beta y^{\beta-1} e^{-2 y^{\beta}}\left(1-e^{-y^{\beta}}\right)^{r-1}\left(e^{-y^{\beta}}\right)^{n-r} d y\right] \\
f_{r: n}(\underline{x})=k \prod_{i=1}^{p} x_{i}^{\alpha_{i}-1} \exp \left(-\sum_{i=1}^{p} x_{i}^{\alpha_{i}}\right)\left[1+\theta \prod_{i=1}^{p}\left(1-2 e^{-x_{i}^{\alpha_{i}}}\right)\left(\frac{2 r-n-1}{n+1}\right)\right]
\end{gathered}
$$

where $k=\prod_{i=1}^{p} \alpha_{i}$

\section{Moments for Multivariate Order Statistics for Trivariate Weibull Distribution}

In this section the Moments for $\mathrm{r}^{\text {th }}$ multivariate order statistics has been derived;

$$
\begin{aligned}
E\left(X_{1 r: n}^{k}, X_{2 r: n}^{k}, \ldots . . X_{p r: n}^{k}\right) & =\int_{0}^{\infty} \int_{0}^{\infty} \ldots . . \int_{0}^{\infty} x_{1}^{k} x_{2}^{k} \ldots \ldots x_{p}^{k} f_{r ; n}\left(x_{1}, x_{2}, \ldots . x_{p}\right) d x_{1} d x_{2} \ldots . d x_{p} \\
E\left(X_{1 r: n}^{k}, X_{2 r: n}^{k}, \ldots . . X_{p r: n}^{k}\right)= & \int_{0}^{\infty} \int_{0}^{\infty} \ldots \ldots \int_{0}^{\infty} x_{1}^{k} x_{2}^{k} \ldots . . x_{p}^{k} k \prod_{i=1}^{p} x_{i}^{\alpha_{i}-1} \exp \left(-\sum_{i=1}^{p} x_{i}^{\alpha_{i}}\right) \\
& {\left[1+\theta \prod_{i=1}^{p}\left(1-2 e^{-x_{i}^{\alpha_{i}}}\right)\left(\frac{2 r-n-1}{n+1}\right)\right] d x_{1} d x_{2} \ldots . . d x_{p} } \\
E\left(X_{1 r: n}^{k}, X_{2 r: n}^{k}, \ldots . . X_{p r: n}^{k}\right)= & \prod_{t=1}^{p} I_{t}+\theta\left(\frac{2 r-n-1}{n+1}\right) \times \prod_{t=1}^{p} M_{t}
\end{aligned}
$$

where $I_{t}=\int_{0}^{\infty} x_{t}^{k} \alpha_{t} x_{t}^{\alpha_{t}-1} e^{-x_{t}^{\alpha_{t}}} d x_{t}$

and $\quad M_{t}=\int_{0}^{\infty} x_{t}^{k} \alpha_{t} x_{t}^{\alpha_{1}-1} e^{-x_{t}^{\alpha_{t}}}\left(1-2 e^{-x_{t}^{\alpha_{t}}}\right) d x_{t}$

Consider $I_{t}$ as:

$$
\begin{aligned}
& I_{t}=\int_{0}^{\infty} x_{t}^{k} \alpha_{t} x_{t}^{\alpha_{t}-1} e^{-x_{t}^{\alpha_{t}}} d x_{1} \\
& I_{t}=\frac{1}{\alpha_{t}^{k}} \int_{0}^{\infty} w_{t}^{k / \alpha_{t}} e^{-w_{t}} d w_{t}=\Gamma\left(k / \alpha_{1}+1\right)
\end{aligned}
$$


Now consider $M_{t}$ as:

$$
\begin{aligned}
M_{t} & =\int_{0}^{\infty} x_{t}^{k} \alpha_{t} x_{t}^{\alpha_{1}-1} e^{-x_{t}^{\alpha_{t}}}\left(1-2 e^{-x_{t}^{\alpha_{t}}}\right) d x_{t} \\
& =\int_{0}^{\infty} w_{t}^{k / \alpha_{1}} e^{-w_{t}}\left(1-2 e^{-w_{t}}\right) d w_{t}=\Gamma\left(k / \alpha_{t}+1\right)\left(\frac{2^{k / \alpha_{t}}-1}{2^{k / \alpha_{t}}}\right)
\end{aligned}
$$

Using values of $I_{1}, I_{2}, \ldots \ldots I_{p} \& M_{1}, M_{2}, \ldots . . M_{p}$ in (5.7.2) we have:

$$
\begin{aligned}
E\left(X_{1 r: n}^{k}, X_{2 r: n}^{k}, \ldots \ldots X_{p r: n}^{k}\right) & =\sqrt{\frac{k}{\alpha_{1}}+1} \sqrt{\frac{k}{\alpha_{2}}+1}, \ldots \ldots \ldots, \sqrt{\frac{k}{\alpha_{p}}+1}+\theta\left(\frac{2 r-n-1}{n+1}\right) \\
& \frac{k}{\alpha_{1}}+1\left(\frac{2^{k / \alpha_{1}}-1}{2^{k / \alpha_{1}}}\right) \cdot \sqrt{\frac{k}{\alpha_{2}}+1}\left(\frac{2^{k / \alpha_{2}}-1}{2^{k / \alpha_{2}}}\right) \ldots . \cdot \frac{k}{\alpha_{p}}+1\left(\frac{2^{k / \alpha_{p}}-1}{2^{k / \alpha_{p}}}\right) \\
E\left(X_{1 r: n}^{k}, X_{2 r: n}^{k}, \ldots \ldots X_{p r: n}^{k}\right) & =\prod_{i=1}^{p} \sqrt{\frac{k}{\alpha_{i}}+1}+\prod_{i=1}^{p} \sqrt{\frac{k}{\alpha_{i}}+1}\left(\frac{2^{k / \alpha_{i}}-1}{2^{k / \alpha_{i}}}\right) \theta\left(\frac{2 r-n-1}{n+1}\right) \\
E\left(X_{1 r: n}^{k}, X_{2 r: n}^{k}, \ldots \ldots X_{p r: n}^{k}\right) & =\prod_{i=1}^{p} \sqrt{\frac{k}{\alpha_{i}}+1}+\theta\left(\frac{2 r-n-1}{n+1}\right) \prod_{i=1}^{p} \sqrt{\frac{k}{\alpha_{i}}+1}\left(\frac{2^{k / \alpha_{i}}-1}{2^{k / \alpha_{i}}}\right)
\end{aligned}
$$

The moments of specific order can be obtained from (6.3).

\section{References}

1. Arnold, B. C., Castillo, E. \& Sarabia, J. M. (2009). Multivariate order statistics via multivariate concomitants. J. Mult, Anal., 100, 946-951.

2. Barakat, H. M. (2009). Multivariate order statistics based on dependent and nonidentically distributed random variables. Journal of Multivariate Analysis, 100, 81-90.

3. Belzunce, F., Ruiz, J. M., \& Ruiz, M. D. C. (2003). Multivariate properties of random vectors of order statistics. Journal of statistical planning and inference, $115,413-424$.

4. David, H. A. \& Nagaraja, H. (2003). Order Statistics. 3rd edn. John Wiley \& Sons, New York.

5. Gumbel, E.J. (1958). Statistics of Extremes, Columbia University Press, New York.

6. Hafeez, M., Kamal, S. \& Shahbaz, M. Q. (2011). The Bivariate Order Statistics for Exponential Distribution. World App. Sci. J., 13(10), 2211-2215.

7. Veena, T. G., Thomas, P. Y. (2008). Characterizations of bivariate distributions by properties of concomitants of order statistics. Statistics and Probability Letters, $78,3350-3354$. 